\title{
Transfer deep learning approach for detecting coronavirus disease in X-ray images
}

\author{
Mohammed Al-Smadi, Mahmoud Hammad, Qanita Bani Baker, Saja Khaled Tawalbeh, \\ Sa'ad A. Al-Zboon \\ College of Computer and Information Technology, Jordan University of Science and Technology, Irbid, Jordan
}

\begin{tabular}{l}
\hline \hline Article Info \\
\hline Article history: \\
Received Sep 9, 2020 \\
Revised Jun 6, 2021 \\
Accepted Jun 16, 2021 \\
\hline
\end{tabular}

Keywords:

CNN

COVID-19 transfer learning

epidemic

Deep learning detecting

\begin{abstract}
Currently, the whole world is fighting a very dangerous and infectious disease caused by the novel coronavirus, called COVID-19. The COVID-19 is rapidly spreading around the world due to its high infection rate. Therefore, early discovery of COVID-19 is crucial to better treat the infected person as well as to slow down the spread of this virus. However, the current solution for detecting COVID-19 cases including the PCR test, CT images, epidemiologically history, and clinical symptoms suffer from high false positive. To overcome this problem, we have developed a novel transfer deep learning approach for detecting COVID-19 based on x-ray images. Our approach helps medical staff in determining if a patient is normal, has COVID-19, or other pneumonia. Our approach relies on pre-trained models including Inception-V3, Xception, and MobileNet to perform two tasks: i) binary classification to determine if a person infected with COVID-19 or not and ii) a multi-task classification problem to distinguish normal, COVID-19, and pneumonia cases. Our experimental results on a large dataset show that the F1-score is $100 \%$ in the first task and 97.66 in the second task.
\end{abstract}

This is an open access article under the CC BY-SA license.

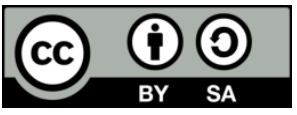

\section{Corresponding Author:}

Mohammed Al-Smadi

College of Computer and Information Technology

Jordan University of Science and Technology

Irbid, 22110, Jordan

Email: masmadi@just.edu.jo

\section{INTRODUCTION}

The whole world currently is fighting the recently discovered infectious disease known as COVID-19. The World Health Organization (WHO) [1] announced the COVID-19 outbreak as a global pandemic on February of 2020. COVID-19 causes a variety of infections related to the respiratory system with no vaccine against this virus so far. The first death caused by this virus reported on January 11 of 2020 . As mentioned in [2], the infection rate of COVID-19 is 3.77, i.e., a sick person can infect about 4 other persons. Due to the fast spread of COVID-19, as of December 9 of 2020, more than 68.3 million people have been infected with COVID-19 and more than 1.5 million persons lost their lives due to this virus in 220 different countries and territories around the world. This virus caused all governments around the world to take severe actions to prevent the spreading of this virus including quarantine, partial and complete lock-down to various cities or even the whole country, moving the education to distance education, moving physical work places to virtual work environments whenever possible, and urging people to do social distancing or better stay at home. 
Several ways have been followed to diagnose the COVID-19 cases. For example, COVID-19 testing kit, positive CT images, epidemiological history, and clinical symptoms (i.e., fever, cough, dyspnea, and pneumonia) [3], [4]. However, relying on the clinical symptoms cannot be generalized since an infected person can carry the virus for few days before the symptoms appears. Moreover, different persons respond differently to the virus based on their health condition and their immune system. However, several factors affected the accuracy of the results obtained from the testing kits such as the quality and the availability of the testing kits as well as the need for retaking the test several times, in some cases, to make sure that the person is tested negative against COVID-19. Therefore, carefully analyzing X-ray images and CT scans give medical staff better understanding of the health status of the patient including if he is being infected by COVID-19, suffer from other pneumonia, or normal [5]. Moreover, carefully analyzing X-rays and CT scans allow medical staff to understand the infected area and the severity of the disease. Unfortunately, determining COVID-19 from other types of pneumonia or even normal using $\mathrm{x}$-ray images is not a trivial process and error-prone for many radiologists and medical staff. To that end, this research tries to help medical staff in accurately detecting COVID-19 in x-ray images using deep neural networks techniques.

In response to this global pandemic, many researchers developed several diagnosing systems depending on deep learning and machine learning techniques. Deep learning techniques are perfect candidate for medical imaging dataset since they can extract important features from the medical images including shape and spatial relation features. In particular, convolutional neural networks (CNNs) provide the best performance regarding feature extraction as well as their ability to improve learning from lowlight images extracted from videos [6]. Furthermore, CNN approaches have been successfully utilized to recognize several diseases, for instance, classifying polyps during colonoscopic videos as well as using x-ray images for diagnosis of pediatric pneumonia [7], [8]. Several features are accredited to identify the viral pathogens based on scans patterns (i.e. CT or x-ray) [9]. The characteristic that distinguishes COVID-19 from others are the bilateral distribution of patchy shadows and ground-glass opacity in the early stages. Moreover, multiple ground glass and infiltrates in lungs will appear in late stages [3]. CNN approaches may help to identify the unique features of COVID-19 pneumonia regarding the difficulty of visual recognition. In this research, we proposed a diagnostic system using deep learning technique based on convolutional neural networks (CNNs) to detect COVID-19 cases. The main contribution of this research can be summarized as:

- Novel transfer learning models; to develop our COVID-19 prediction system, we have developed a novel transfer deep learning approach. Our transfer learning relies on 3 state-of-the-art CNN models that have been pre-trained on ImageNet dataset followed by global average pooling to extract discriminative features, then batch normalization and dropout to prevent the overfitting.

- Dataset; we have collected a dataset consisting of 7,800 x-ray images from different sources. These images contains x-ray for patients with COVID-19, normal, and patients with other pneumonia. To enlarge our dataset, we have applied various data augmentation techniques and generated 499,200 labeled $\mathrm{X}$-ray images. We make this dataset available to other researchers and practitioners on demand.

- Comprehensive study; we provide a comprehensive study about the available datasets for COVID-19 research as well as the related work effort in detecting COVID-19 using machine learning techniques.

To develop and evaluate our approach, we have used 7,800 X-ray images and focused on two main tasks. The first task is to develop a binary classifier that can distinguish between COVID-19 or non COVID $\mathrm{X}$-ray image. The second task is to build a multi-class classifier that can determine if an X-ray image contains COVID-19, other pneumonia, or normal image. Our transfer deep learning model utilized 3 stat-of-the-art models, Inception-V3, Xception, and MobileNet. Using out dataset after data augmentation, our approach achieved $100 \%$ accuracy and $100 \%$ F-score on the first task. Regarding the second task, our best model achieved accuracy of $97.37 \%$ and an F-score of $97.66 \%$.

The rest of this paper is organized as follows; section 2 discuss the related research effort to our research and provide a detailed discussion about the available datasets of COVID-19. Section 3 describes our method in mode details and section 4 shows the experimental results of our approach. Section 5 discusses the results. Finally, our paper concludes with avenue of future directions in section 6.

\section{RELATED WORK}

Due to the global pandemic caused by the novel coronavirus, COVID-19, many researchers in all fields worked very hard to study and investigate this virus and its effect of the humanity. In the machine learning filed, some researchers tried to collect datasets, textual and images, to make them available to others. While others tried to develop new techniques to detect the COVID-19 accurately. This section (1) discusses the related research efforts in the machine learning field, section 2.1 as well as (2) discussing the available COVID-19 datasets in section 2.2. 


\subsection{Machine learning based techniques}

Although the work on the medical images is related to our work [10]-[12], due to the space limitation, in this section we will discuss the related research efforts in detecting COVID-19 using machine learning techniques. Yan et al. [13] introduced an XGBoost classifier to predict the prognostic state's severe of COVID-19 infection using clinical data in Wuhan, China. In addition, Pal et al. [14] introduced an LSTM model to predict the long duration outbreak caused by COVID-19 and how the risk affects the countries so they can take preventive steps earlier. On the other hand, Matteo et al. [15] collected large dataset obtained from the social media platforms (e.g., Twitter, YouTube, Facebook) related to the COVID-19 and analyzed them. Wang et al. [16] introduced a diagnostic evaluation using a transfer learning based on deep learning approach (Inception network) using CT images to detect the COVID-19. Similarity, Sethy et al. [17] proposed several deep neural networks based on SVM using deep features to detect COVID-19 in X-ray images. Shan et al. [18] introduced an automatic segmentation based on deep learning to specify the infection regions of the COVID-19 based on their shapes, volumes, and percentage of infection (POI) relying on chest CT scans.

Maghdid et al. [19] presented the idea of proposing AI tools for COVID-19 diagnosis as well as performing deep learning system using CT and x-ray images. Similarly, Xu et al. [20] studied the possibility of proposing deep learning approaches to be used as a diagnostic system to predict COVID-19 or other pneumonia using CT scans. Gozes et al. [21] proposed automated CT image analysis tool aims to detect and track if the patient infected of COVID-19 disease or not. Li et al. [22] proposed deep learning approach called COVNet (COVID-19 detection neural network) that is be able to obtain visual features from the volumetric CT scans and distinguish between COVID-19 and other pneumonia. Bukhari et al. [23] proposed transfer learning using pre-trained ImageNet aims to avoid lack of dataset issue to detect one of the three classes COVID-19, pneumonia, or normal. Similarly, Apostolopoulos et al. [24] proposed transfer learning adaption that performs CNN from scratch called Mobile Net to detect types of pneumonia. Narin et al. [25] proposed 3 deep CNN models to classify a balanced dataset of x-ray images to COVID-19 or nonCOVID. Jaiswal et al. [26] proposed deep learning model to identifying pneumonia types. Arora et al. [27] presented an analysis study using deep learning regarding COVID-19. Farooq and Hafeez [28] introduced an enhancement of the previous models to detect pneumonia cases using fine-tuning ResNet50. However, Alqudah et al. [29] proposed hybrid approach based on CNN models to detect COVID-19 in earlier stages. Alom et al. [30] introduced end-to-end system to detect COVID-19 and select the infected area. Similarly, Hammoudi et al. [31] proposed an automatic CNN approach that aims to detect the estimation of infection rate using $\mathrm{x}$-ray images. Rahmatizadeh et al. [32] proposed an AI-based decision-making system aims to help and manage the COVID-19 ICU patients. Moreover, Shi et al. [33] and Nguyen [34] introduced comprehensive reviews of deep learning approaches that used to detect and specify the infected area as well as how the AI can help to reduce the COVID-19 infection, spread, and detection.

All of the aforementioned research efforts supports that deep learning is a useful tool for detecting COVID-19 from various sources in order to help medical staff in diagnosing patients as well as reducing the spread of this virus. Our research is similar to these research effort but significantly different. We have leveraged transfer learning technique to make our approach faster and requires less computational specifications. We have collected x-ray images systematically from different data sources and applied various sophisticated data augmentation techniques to increase the performance of our approach.

\subsection{COVID-19 available datasets}

This section lists the main COVID-19 datasets to help other researchers and practitioners. Many datasets present textual information about the COVID-19 obtained from the Twitter platform including [35], with over 110M tweets [36], with over 40M tweets [37], with over 150M tweets [38], and with over 75M tweets [39]. Moreover, provide dataset and kernels to analyze COVID-19 related tweets [40]-[42].

For real time research, this humanitarian data exchange [43] broadcasts a live map for the COVID-19. Interactive visualizations presented by Nextstrain [44]. Moreover, Kaggle [45] provided chest X-ray images used for Pneumonia detection with 5,863 images. Similarly, Cohen et al. [46] presented chest x-ray and CT images to detect COVID-19, MERS, SARS, and ARDS. Yang et al. [47] built a CT images dataset to detect COVID-19. MedSeq [48] provided a COVID-19 CT segmentation dataset. Radiopaedia [49] aims to create the best radiology reference for the world for COVID-19 positive cases. Several resources are rich with information about the COVID-19 and it is updated frequently such as [50] and [51]. Finally, this website [52] provides a COVID-19 epidemiological dataset.

\section{RESEARCH METHOD}

\subsection{Dataset preparation}

To collect X-ray images for patients with positive COVID-19, we have collected x-rays images from the datasets mentioned in section 2.2. We also collected x-ray images for patients with COVID-19 diagnosed

\footnotetext{
Transfer deep learning approach for detecting coronavirus disease (COVID-19)... (Mohammed Al-Smadi)
} 
clinically, using symptoms such as cough, sputum, fever, dyspnea, or pleuritic chest pain, from [53]-[57]. Regarding the non COVID-19 images, we obtained x-rays images from Kaggle competitions that contain $\mathrm{X}$-ray images for normal and pneumonia chest images. The ages of the patients in our dataset are between 25 and 85. Some images represent the $\mathrm{x}$-ray for the same patient taken on different dates.

Figure 1 depicts examples of the x-ray images for patients with COVID-19, the images in the above row, and normal patients, the images in the below row, in our dataset. Similarly, Figure 2 shows examples of the images in our dataset used for the second task, the multi-class classification task. The figure shows x-ray images for normal patients (the lower row), patients with COVID-19 (the middle row), and patients with other types of pneumonia (the upper row). Table 1 summarizes our dataset including the number of $\mathrm{x}$-ray images for training and testing.

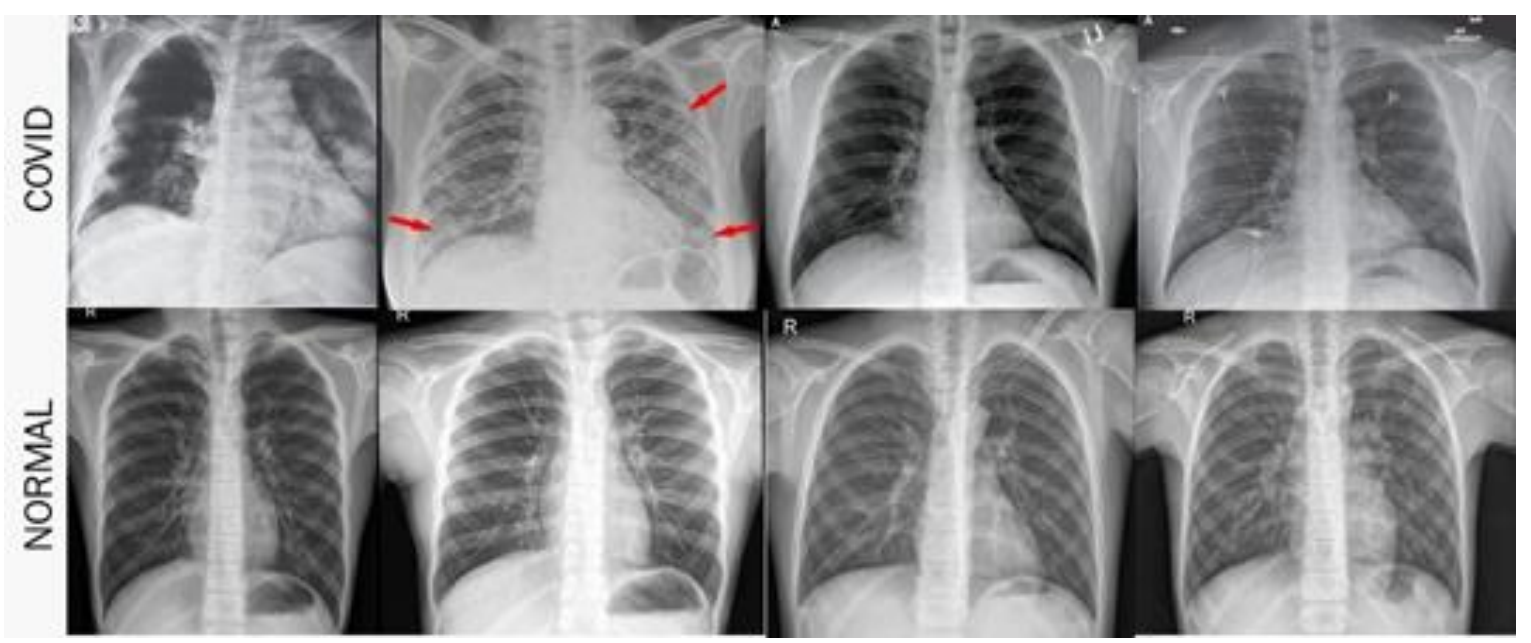

Figure 1. Sample x-ray images from our dataset used for the first task

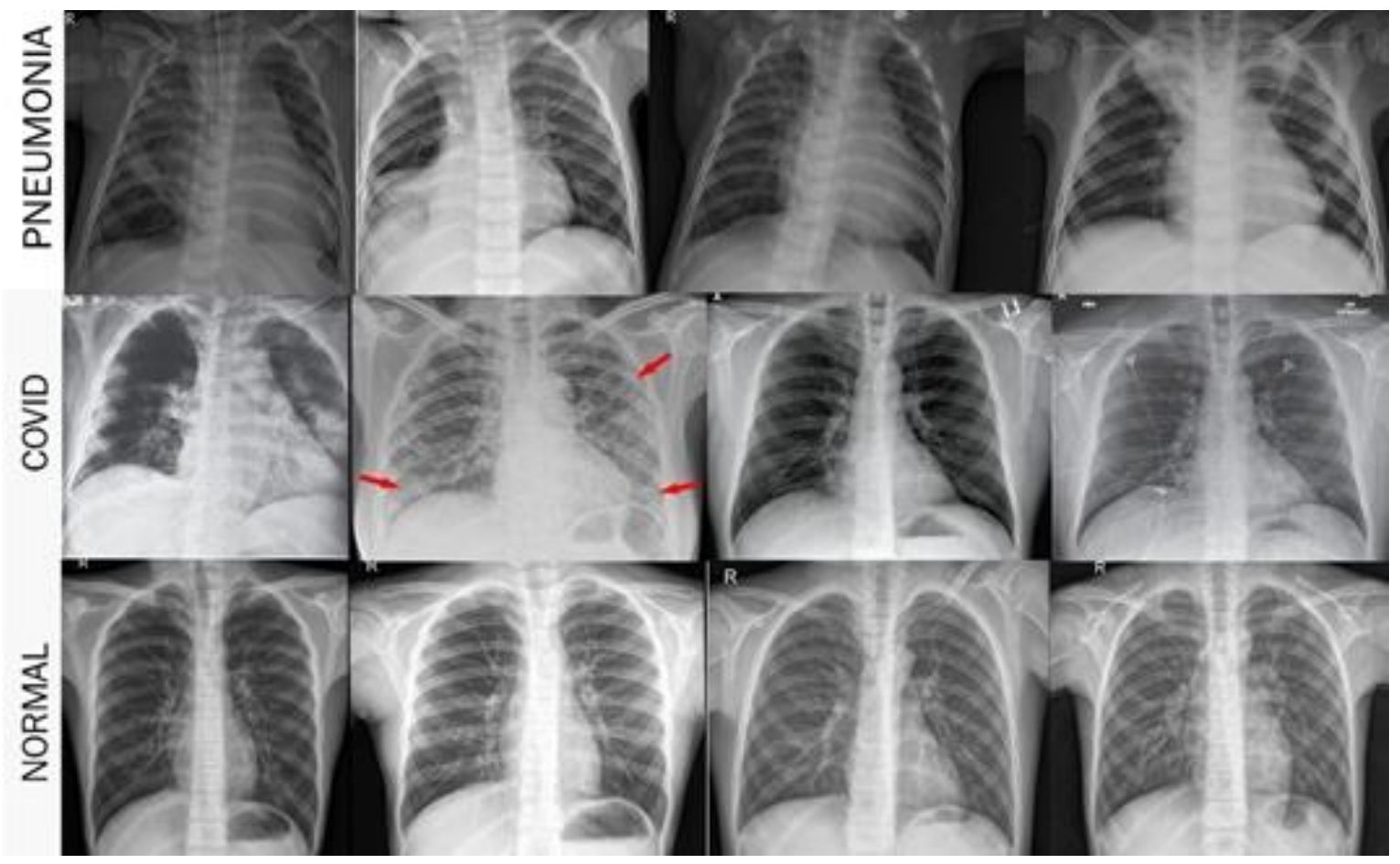

Figure 2. Sample $\mathrm{x}$-ray images from our dataset used for the second task 
Table 1. Summary of the dataset used for training and evaluating our transfer deep learning model

\begin{tabular}{ccccc}
\hline Files & COVID-19 & Non COVID-19 & Pneumonia & Total \\
\hline Train & 865 & 1,957 & 3,418 & 6,240 \\
Test & 216 & 489 & 855 & 1,560 \\
Total & 1,081 & 2,446 & 4,273 & 7,800 \\
\hline
\end{tabular}

\subsection{Data augmentation}

The data augmentation process increases the number and the diversity of the dataset and hence reduces the overfitting and the memorization problems of machine learning models. Moreover, since deep learning models require large dataset, this technique is crucial for building stable and generalizable deep learning models. To that end, we have applied various data augmentation techniques, as presented in Table 2, to enlarge our dataset. The total number of images after the data augmentation is 499,200 labeled images.

Table 2. Advanced augmentation types that have used and their parameters

\begin{tabular}{|c|c|}
\hline Augmentation Type & Description and Parameters \\
\hline Scale & Re-scale the images outward or inward. \\
\hline Shearing or Shear Mapping & Displaced each pixel in the vertical direction by -16 proportional to +16 degrees. \\
\hline Elastic and Perspective Transformation & $\begin{array}{l}\text { Elastic: use the random strengths to move pixels locally around. Perspective: creates a } \\
\text { different image captured from different camera viewpoints alpha }=(0.5,3.5) \text {, sigma }=0.25\end{array}$ \\
\hline Gaussian Blur & $\begin{array}{l}\text { Produces a blurry image, the edges become sharper using high contrast filter. Blur } \\
\text { images with a sigma between }(0,3.0)\end{array}$ \\
\hline Average Blur & $\begin{array}{l}\text { Computing a simple median over neighborhoods. Blur image using local means with } \\
\text { kernel sizes between }(3,5)\end{array}$ \\
\hline Median Blur & $\begin{array}{l}\text { Gets rid of the small noise in the images which caused by computing the median values } \\
\text { over neighborhoods. } \\
\text { blur image using local medians with kernel sizes between }(3,5)\end{array}$ \\
\hline Emboss & $\begin{array}{l}\text { Overlays the image with the original images by tuning alpha between } 0.0 \text { and } 1.0 \text {. } \\
\text { alpha }=(0,1.0) \text {, strength }=(0,2.0)\end{array}$ \\
\hline Additive Gaussian Noise & $\begin{array}{l}\text { It mitigates overfitting and a dishonest procedure for real valued inputs by adding noise } \\
\text { sampled to the images. } l o c=0 \text {, scale }=(0.0,0.01 * 255) \text {, per channel }=0.5\end{array}$ \\
\hline
\end{tabular}

\subsection{Transfer learning approach}

This section describes our transfer deep learning approach of detecting COVID-19 images using our transfer learning model for the sake of helping medical staff accurately and confidently diagnosis COVID-19 in medical images and hence better treating the patients as early as possible. Transfer learning technique has improved the performance of image classification and computer vision research as noted in Litjens et al. [58] work. The main idea of transfer learning is to re-use a previously trained model on similar domain instead of re-training a new model from scratch. Hence boosting the performance (in terms of time and accuracy) of the transfer learning model. Moreover, transfer learning allows us to use a previously trained model that might be trained on a high specifications computing device that we might not have or above our budget. For example, the ImageNet dataset, introduced by Russakovsky et al. [59], contains thousands of images. Many neural network models have been trained on the ImageNet dataset including VGG, Inception, Xception, and ResNet.

To address our problem, we have developed a CNN approach based on transfer learning to solve the two image classification tasks of our COVID-19 detection problem. In our approach, we have utilized two state-of-the-art CNN models: the Inception model introduced by Szegedy et al. [60] and the Xception model proposed by Chollet [61]. Figure 3 depicts the architecture of our approach which starts by leveraging various data augmentation techniques (recall section 3.2.) then the resulted images fed to the pre-trained model. After that, a global average pooling 2D is performed to extract the discriminative features from the images to use them in the next layers. Next, the batch normalization layer is performed to standardize the inputs for each mini batch and to stabilize the learning process. The flatten layer is applied to convert the input data to a 1D array and fed it to the dense layer with 128 neurons, then to the dropout layer with a dropout rate of 0.5 . Finally, the prediction layer consists of dense of 1 for the COVID-19 binary image classification task and dense of 3 for the second multi-task classification task with a softmax activation function for input classification. 


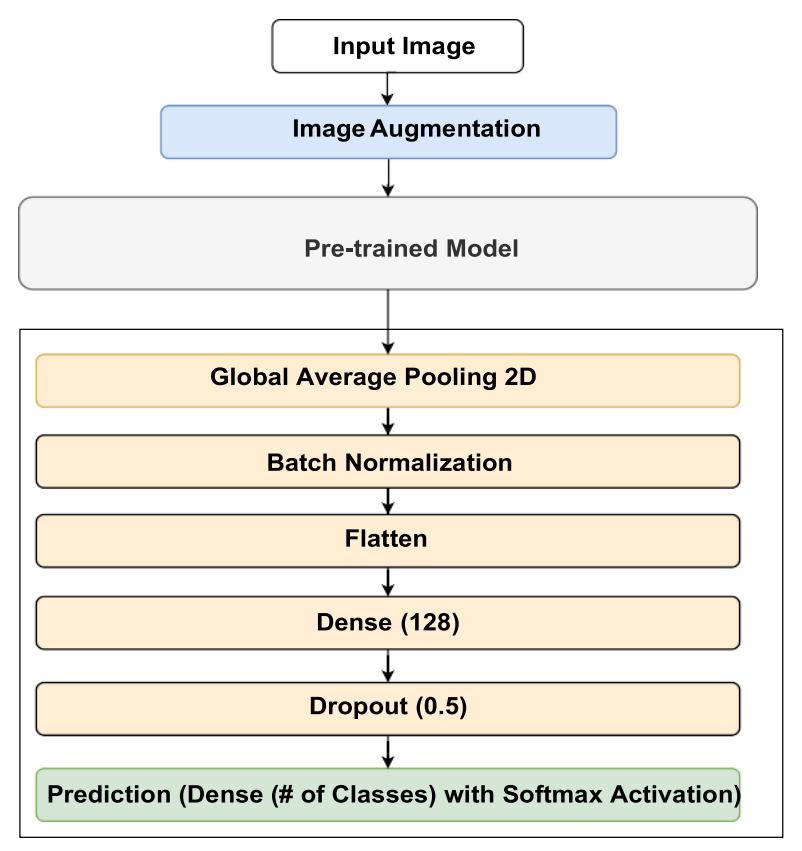

Figure 3. The architecture of our transfer deep learning approach

\section{EXPERIMENTAL EVALUATION}

\subsection{Performance metrics}

Several standard measures have been used to evaluate the performance of our models.

$$
\begin{aligned}
& \text { Accuracy }=\frac{T P+T N}{T P+F P+T N+F N} \\
& F 1_{\text {Score }}=\frac{2 * \text { Precision } * \text { Recall }}{\text { Precision }+ \text { Recall }}
\end{aligned}
$$

Where Precision: represents the number of cases that the model predicted correctly out of the overall predicted cases. The precision measure can be computed using (3):

$$
\text { Precision }=\frac{T P}{T P+F P}
$$

Recall: represents the number of cases that the model predicted correctly out of the overall cases in the dataset. The recall measure can be computed using (4):

$$
\text { Recall }=\frac{T P}{T P+F N}
$$

- True Positives (TP): can be defined as the cases in which the predicted case diagnosed as COVID-19 and the actual diagnose is COVID-19.

- True Negatives (TN): can be defined as the cases in which the predicted case diagnosed as nonCOVID-19 and the actual case diagnosed as nonCOVID.

- False Positives (FP): can be defined as the cases in which the predicted case diagnosed as COVID-19 whereas the actual case diagnosed as nonCOVID.

- False Negatives (FN): can be defined as the cases in which the predicted case diagnosed as nonCOVID-19 where as the actual case diagnosed as COVID-19.

\subsection{Experimental results}

In order to evaluate the developed transfer learning approach, we collected COVID-19 dataset from different resources that aims to solve both problems. Several simple and advanced augmentation techniques are used to increase the number of the training dataset (recall section 3.2.). Table 3 summarizes the 
evaluation results of our transfer deep learning models using 3 different pretrained models, the Inception-V3, the Xception, and the MobileNet models. Regarding the first task, the binary classification task, Table 3 shows that our model with 3 different pre-trained models achieved the perfect score in the accuracy as well as in the F1-Score, i.e., $100 \%$. On the other hand, regarding the multi classification task, Table 3 shows that our model achieved the best results using the Xception pre-trained model with $97.37 \%$ accuracy and $97.66 \%$ F1Score. These high accurate results of our models make us confident that our developed models can be used by medical staff to help them in diagnosing COVID-19 cases accurately and efficiently.

Table 3. Evaluation results of our proposed approach using 3 different pre-trained models using x-ray images dataset for both tasks, the binary and the multi-class classifications

\begin{tabular}{ccccc}
\hline & \multicolumn{2}{c}{ Binary Classification } & \multicolumn{2}{c}{ Multi-Class Classification } \\
Model & Accuracy & F1-Score & Accuracy & F1-Score \\
\hline Inception-V3 & $100 \%$ & $100 \%$ & $97.12 \%$ & $97.47 \%$ \\
Xception & $100 \%$ & $100 \%$ & $97.37 \%$ & $97.66 \%$ \\
MobileNet & $100 \%$ & $100 \%$ & $96.06 \%$ & $96.47 \%$ \\
\hline
\end{tabular}

\section{DISCUSSION}

Several techniques were applied to avoid the problem of overfitting in the model training as follows: i) data augmentation was applied to the dataset and the resulted data were used to train the model; ii) the callback function of Keras "EarlyStopping" [62] is used to stop the model training when the computed validation loss value is not improving during training epochs; and iii) regulation techniques such as Batch Normalization [63] and Dropout [64] were used to reduce the model overfitting and enhance the model learning capabilities.

As depicted in Figure 4, the model was trained without overfitting. Because of the techniques used to reduce overfitting, both loss values of train loss and the validation loss declined together during model training without having large gaps between their computed values over training epochs. In order to validate the proposed model achieved results, an ablation analysis was conducted on the results of the best performing model (Xception+data augmentation+Transfer Learning). As presented in Table 4, training the model without augmented learning decreased the results for binary classification in Task1 with $4.92 \%$ for the F1-score, and for the multi-class classification in Task2 with $4.78 \%$. Ablating the transfer learning and training the Xception model alone on the augmented dataset has a higher influence on the results with a decrease of $6.13 \%$ for binary classification in Task 1 and a decrease of $9.21 \%$ for the multi-class classification in Task2. It also can be noticed that the ablation of both data augmentation and transfer learning has the highest decrease on the tasks results with $11.12 \%$ for binary classification in Task 1 and a decrease of $16.54 \%$ for the multi-class classification in Task2. Ablation results show the influence of the data augmentation techniques used on enhancing the results as well as the strength of the transfer learning model we applied to achieve the tasks requirements.

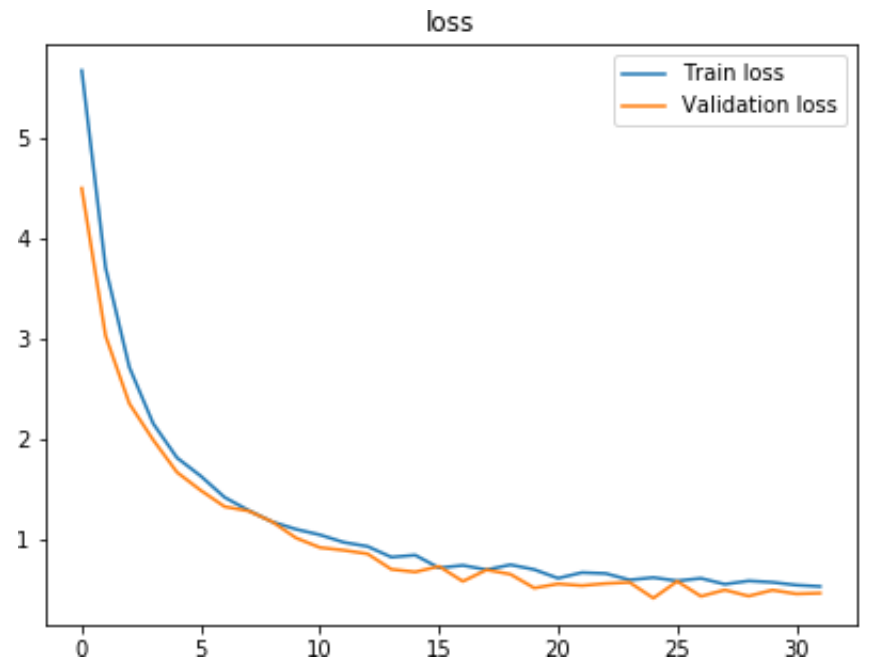

Figure 4. Loss values for the training and validation during each training epoch for the best achieving model 
Table 4. Results for the ablation analysis for the Xception + transfer learning model

\begin{tabular}{ccccc}
\hline & \multicolumn{2}{c}{ Binary Classification } & \multicolumn{2}{c}{ Multi-Class Classification } \\
\hline Ablated Features & F1-Score & Difference & F1-Score & Difference \\
without data augmentation & $95.08 \%$ & $4.92 \%$ & $92.88 \%$ & $4.78 \%$ \\
without transfer learning & $93.87 \%$ & $6.13 \%$ & $88.45 \%$ & $9.21 \%$ \\
without transfer learning + data augmentation & $88.88 \%$ & $11.12 \%$ & $81.12 \%$ & $16.54 \%$ \\
Xception + data augmentation + Transfer Learning & $100 \%$ & - & $97.66 \%$ & - \\
\hline
\end{tabular}

\section{CONCLUSION}

In this research, we have developed a transfer deep learning approach to detect if a patient has a COVID-19 or not using x-rays images. We have leveraged state-of-the-art CNN models (Inception-V3, the Xception, and the MobileNet) as pre-trained models. All of these models have been trained on the ImageNet dataset and they achieved promising results. Our transfer learning approach enhances the performance of our models and increases their accuracy and efficiency. In this research, we focused on two tasks, the first task is a binary classification task in which the model needs to detect if a given $\mathrm{x}$-ray image shows that the patient has COVID-19 or not. In the second task, the model has been trained to distinguish between COVID-19, normal, or pneumonia x-rays. Our experimental evaluations show that our models achieved high accuracy in both tasks, $100 \%$ in the first task and $97.66 \%$ on the second task. These high accuracy proofs that our models can be used by medical staff to diagnose patients with COVID-19 using their x-ray images. In the future, we will develop a transfer deep learning models to predict the stage and the severity of the COVID-19 from $\mathrm{x}$-ray images.

\section{ACKNOWLEDGEMENTS}

This research is partially funded by Jordan University of Science and Technology, Research Grant Numbers: 20200145 and 20190306.

\section{REFERENCES}

[1] WHO, "World health organization (who)," 1948. [Online]. Available: https://www.who.int.

[2] Y. Yang et al., "Epidemiological and clinical features of the 2019 novel coronavirus outbreak in china," medRxiv, 2020, doi: 10.1101/2020.02.10.20021675.

[3] D. Wang et al., "Clinical Characteristics of 138 Hospitalized Patients With 2019 Novel Coronavirus-Infected Pneumonia in Wuhan, China," JAMA, vol. 323, no. 11, pp. 1061-1069, 2020, doi: 10.1001/jama.2020.1585.

[4] C. Huang et al., "Clinical features of patients infected with 2019 novel coronavirus in wuhan, china," The Lancet, vol. 395, no. 10223, pp. 497-506, 2020, doi: 10.1016/S0140-6736(20)30183-5.

[5] D. K. W. Chu et al., "Molecular Diagnosis of a Novel Coronavirus (2019-nCoV) Causing an Outbreak of Pneumonia," Clinical Chemistry, vol. 66, no. 4, pp. 549-555, 2020, doi: 10.1093/clinchem/hvaa029.

[6] P. Go'mez, M. Semmler, A. Schu"tzenberger, C. Bohr, and M. Do"llinger, "Low-light image enhancement of highspeed endoscopic videos using a convolutional neural network," Medical and biological engineering and computing, vol. 57, no. 7, pp. 1451-1463, 2019, doi: 10.1007/s11517-019-01965-4.

[7] J. Choe et al., "Deep learning-based image conversion of CT reconstruction kernels improves radiomics reproducibility for pulmonary nodules or masses," Radiology, vol. 292, no. 2, pp. 365-373, 2019, doi: 10.1148/radiol.2019181960.

[8] D. S. Kermany et al., "Identifying medical diagnoses and treatable diseases by image-based deep learning," Cell, vol. 172, no. 5, pp. 1122-1131, 2018, doi: 10.1016/j.cell.2018.02.010.

[9] H. J. Koo, S. Lim, J. Choe, S.-H. Choi, H. Sung, and K.-H. Do, "Radiographic and ct features of viral pneumonia," Radiographics, vol. 38, no. 3, pp. 719-739, 2018, doi: 10.1148/rg.2018170048.

[10] P. Guo, A. Evans, and P. Bhattacharya, "Nuclei segmentation for quantification of brain tumors in digital pathology images," International Journal of Software Science and Computational Intelligence (IJSSCI), vol. 10, no. 2, pp. 36-49, 2018, doi: 10.4018/IJSSCI.2018040103.

[11] O. Dorgham et al., "Enhancing the security of exchanging and storing dicom medical images on the cloud," International Journal of Cloud Applications and Computing (IJCAC), vol. 8, no. 1, pp. 154-172, 2018, doi: 10.4018/IJCAC.2018010108.

[12] A. Ghoneim, G. Muhammad, S. U. Amin, and B. Gupta, "Medical image forgery detection for smart healthcare," IEEE Communications Magazine, vol. 56, no. 4, pp. 33-37, 2018, doi: 10.1109/MCOM.2018.1700817.

[13] L. Yan et al., "Prediction of criticality in patients with severe covid-19 infection using three clinical features: a machine learning-based prognostic model with clinical data in Wuhan," medRxiv, 2020, doi: 10.1101/2020.02.27.20028027.

[14] R. Pal, A. A. Sekh, S. Kar, and D. K. Prasad, "Neural network based country wise risk prediction of covid-19," Applied Sciences, vol. 10, no. 18, 2020, Art. no. 6448, doi: 10.3390/app10186448. 
[15] M. Cinelli et al., "The covid-19 social media infodemic," Scientific Reports, vol. 10, no. 1, pp. 1-10, 2020, Art. no. 16598, doi: 10.1038/s41598-020-73510-5.

[16] S. Wang et al., "A deep learning algorithm using ct images to screen for corona virus disease (covid-19)," medRxiv, 2020, doi: 10.1101/2020.02.14.20023028.

[17] P. K. Sethy, S. K. Behera, P. K. Ratha, P. Biswas, "Detection of coronavirus disease (covid-19) based on deep features and Support Vector Machine," International Journal of Mathematical, Engineering and Management Sciences, vol. 5, no. 4, pp. 643-651, 2020, doi: 10.33889/IJMEMS.2020.5.4.052.

[18] F. Shan et al., "Lung infection quantification of covid-19 in ct images with deep learning," ArXiv, abs/2003.04655, 2020.

[19] H. S. Maghdid, A. T. Asaad, K. Z. Ghafoor, A. S. Sadiq, and M. K. Khan, "Diagnosing covid-19 pneumonia from $\mathrm{x}$-ray and ct images using deep learning and transfer learning algorithms," Proceedings Volume 11734, Multimodal Image Exploitation and Learning 2021, vol. 11734, 2021, Art. no. 117340E, doi: 10.1117/12.2588672.

[20] X. Xu et al., "A Deep learning system to screen coronavirus disease 2019 pneumonia," Engineering, vol. 6, no. 10, pp. 1122-1129, 2020, doi: 10.1016/j.eng.2020.04.010.

[21] O. Gozes et al., "Rapid ai development cycle for the coronavirus (covid-19) pandemic: Initial results for automated detection and patient monitoring using deep learning ct image analysis," arXiv preprint arXiv:2003.05037, 2020.

[22] L. Li et al., "Artificial intelligence distinguishes covid-19 from community acquired pneumonia on chest CT," Radiology, 2020, Art. no. 200905, doi: 10.1148/radiol.2020200905.

[23] S.U. K. Bukhari, S. S. K. Bukhari, A. Syed, S. S. H. Shah, "The diagnostic evaluation of convolutional neural network (cnn) for the assessment of chest x-ray of patients infected with covid-19," medRxiv, 2020, doi: 10.1101/2020.03.26.20044610.

[24] I. Apostolopoulos, S. Aznaouridis, and M. Tzani, "Extracting possibly representative covid-19 biomarkers from xray images with deep learning approach and image data related to pulmonary diseases," Journal of Medical and Biological Engineering, vol. 40, pp. 462-469, 2020, doi: 10.1007/s40846-020-00529-4.

[25] A. Narin, C. Kaya, and Z. Pamuk, "Automatic detection of coronavirus disease (covid-19) using x-ray images and deep convolutional neural networks," Pattern Analysis and Applications, pp. 1-14, 2021, doi: 10.1007/s10044-02100984-y.

[26] A. K. Jaiswal, P. Tiwari, S. Kumar, D. Gupta, A. Khanna, and J. J. P. C. Rodrigues, "Identifying pneumonia in chest X-rays: A deep learning approach," Measurement, vol. 145, pp. 511-518, 2019, doi: 10.1016/j.measurement.2019.05.076

[27] K. Arora, A. S. Bist, S. Chaurasia, and R. Prakash, "Analysis of deep learning techniques for covid-19 detection," International Journal of Scientific Research In Engineering And Management (IJSREM), vol. 4, no. 4, pp. 1-5, 2020.

[28] M. Farooq and A. Hafeez, "Covid-resnet: A deep learning framework for screening of covid19 from radiographs," 2020, arXiv preprint arXiv:2003.14395.

[29] A. M. Alqudah, S. Qazan, H. H. Alquran, I. Abuqasmieh, and A. Alqudah, "Covid-19 detection using x-ray images and artificial intelligence hybrid systems," Biomedical Signal and Image Analysis and Project; Biomedical Signal and Image Analysis and Machine Learning Lab: Boca Raton, FL, USA, 2019, doi: 10.5455/jjee.204-158531224.

[30] Md Z. Alom, M. M. S. Rahman, M. S. Nasrin, T. M. Taha, and V. K. Asari, "COVID MTNet: COVID-19 Detection with Multi-Task Deep Learning Approaches," arXiv e-prints, 2020.

[31] K. Hammoudi et al., "Deep learning on chest x-ray images to detect and evaluate pneumonia cases at the era of covid-19," 2020, ArXiv preprint arXiv:2004.03399.

[32] S. Rahmatizadeh, S. V.-Haghi, and A. Dabbagh, "The role of artificial intelligence in management of critical covid19 patients," Journal of Cellular and Molecular Anesthesia, vol. 5, no. 1, pp. 16-22, 2020.

[33] F. Shi et al., "Review of artificial intelligence techniques in imaging data acquisition, segmentation and diagnosis for covid-19," IEEE Reviews in Biomedical Engineering, vol. 14, pp. 4-15, 2020, doi: 10.1109/RBME.2020.2987975.

[34] T. T. Nguyen, "Artificial intelligence in the battle against coronavirus (covid-19): a survey and future research directions," 2020, arXiv preprint arXiv:2008.07343.

[35] Tweet Binder, "Covid 19-twitter evolution," [Online]. Available: https://www.tweetbinder.com/blog/covid-19coronavirus-twitter/

[36] R. Lamsal, "Covid-19 tweets dataset," IEEE data Port, 2020, doi: 10.21227/781w-ef42.

[37] J. M. Banda and R. Tekumalla, "A Twitter Dataset of 40+ million tweets related to COVID-19," This dataset will be updated bi-weekly, 2020.

[38] J. M. Banda et al., "A Twitter Dataset of 150+ million tweets related to COVID-19, March 2020," This dataset will be updated bi-weekly at least with additional tweets, look at the github repo for these updates, 2020.

[39] Panacea Lab, "Covid-19 twitter chatter dataset," [Online]. Available: http://www.panacealab.org/covid19/

[40] Kaggle, "Tweets using hashtags with COVID19," [Online]. Available: https://www.kaggle.com/discussion/232558.

[41] Kaggle, "Day level information on covid-19 affected cases," [Online]. Available: https://www.kaggle.com/sudalairajkumar/novel-corona-virus-2019-dataset

[42] Kaggle, "Covid-19 open research dataset challenge (cord-19)," [Online]. Available: https://www.kaggle.com/alleninstitute-for-ai/CORD-19-research-challenge

[43] Humanitarian Data Exchange (HDX), "Novel covid-19 time series data," [Online]. Available: https://data.humdata.org/dataset/novel-coronavirus-2019-ncov-cases

[44] Nextstrain, "Genomic epidemiology of novel coronavirus," [Online]. Available: https://nextstrain.org/ncov/global 
[45] Kaggle, “Chest x-ray images [Online]. https://www.kaggle.com/paultimothymooney/chest-xray-pneumonia.

[46] J. P. Cohen, P. Morrison, and L. Dao, "Covid-19 image data," arXiv 2003.11597, 2020.

[47] X. Yang, X. He, J. Zhao, Y. Zhang, S. Zhang, and P. Xie, "Covid-ct dataset: a ct image dataset about covid-19," arXiv preprint arXiv:2003.13865, 2020.

[48] Medical Segmentation, "Covid-19 ct segmentation," [Online]. Available: https://www.eibir.org/covid-19-imagingdatasets/

[49] Radiopaedia, "Radiopaedia.org," 2005. [Online]. Available: https://radiopaedia.org/

[50] Montreal “AI. Covid-19-resources," [Online]. Available: https://mila.quebec/en/covid-19/

[51] TrainingData.io, "Covid-19 radiology dataset (chest xray \& ct) for annotation \& collaboration," [Online]. Available: https://towardsdatascience.com/covid-19-imaging-dataset-chest-xray-ct-for-annotation-collaboration$5 f 6 e 076 f 5 f 22$

[52] Data and Resources, "Covid-19 epidemiological data," [Online]. Available: https://sites.google.com/view/datascience-covid-19/

[53] L. T. Phan et al., "Importation and human-to-human transmission of a novel coronavirus in vietnam," New England Journal of Medicine, vol. 382, no. 9, pp. 872-874, 2020, doi: 10.1056/NEJMc2001272

[54] S. Salehi, A. Abedi, S. Balakrishnan, and G. Ali, "Coronavirus disease 2019 (covid-19): a systematic review of imaging findings in 919 patients," American Journal of Roentgenology, vol. 215, no. 1, pp. 87-93, 2020, doi: 10.2214/AJR.20.23034.

[55] Z. Y. Zu et al., "Coronavirus disease 2019 (covid-19): A perspective from china," Radiology, vol. 296, no. 2, pp. E15-E25, 2020, Art. no. 200490, doi: 10.1148/radiol.2020200490.

[56] W. Kong and P. P. Agarwal, "Chest imaging appearance of covid-19 infection," Radiology: Cardiothoracic Imaging, vol. 2, no. 1, 2020, Art. no. e200028, doi: 10.1148/ryct.2020200028.

[57] H. Y. F. Wong et al., "Frequency and distribution of chest radiographic findings in covid-19 positive patients," Radiology, vol. 296, no. 2, pp. E72-E78, 2020, Art. no. 201160, doi: 10.1148/radiol.2020201160.

[58] G. Litjens et al., "A survey on deep learning in medical image analysis," Medical image analysis, vol. 42, pp. 60-88, 2017, doi: 10.1016/j.media.2017.07.005.

[59] O. Russakovsky et al., "Imagenet large scale visual recognition challenge," International journal of computer vision, vol. 115, no. 3, pp. 211-252, 2015, doi: 10.1007/s11263-015-0816-y.

[60] C. Szegedy, V. Vanhoucke, S. Ioffe, J. Shlens, and Z. Wojna, "Rethinking the inception architecture for computer vision," Proceedings of the IEEE conference on computer vision and pattern recognition, 2016, pp. 2818-2826

[61] F. Chollet, "Xception: Deep learning with depthwise separable convolutions," 2017 IEEE Conference on Computer Vision and Pattern Recognition (CVPR), 2017, pp. 1251-1258, doi: 10.1109/CVPR.2017.195.

[62] Keras, "Keras api," [Online]. Available: https://keras.io/callbacks/

[63] S. Ioffe and C. Szegedy, "Batch normalization: Accelerating deep network training by reducing internal covariate shift," Proceedings of the 32nd International Conference on Machine Learning, vol. 37, 2015, pp. 448-456.

[64] N. Srivastava, G. Hinton, A. Krizhevsky, I. Sutskever, and R. Salakhutdinov, "Dropout: a simple way to prevent neural networks from overfitting," The journal of machine learning research, vol. 15, no. 56, pp. 1929-1958, 2014. 